
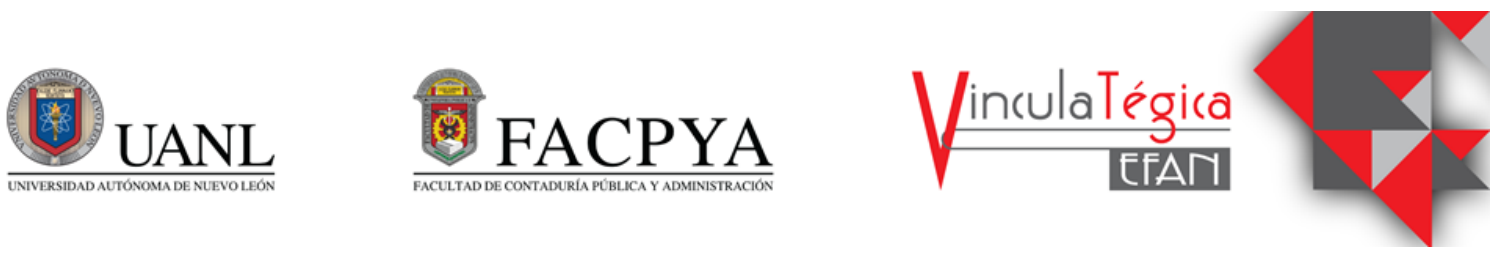

\title{
Franquicia como opción ante la pandemia: un estudio de acciones operativas
}

\author{
Ronald Santos Cori $^{1}$ \\ ${ }^{1}$ UANL FACPYA (México), correo: ronald.santoscr@uanl.edu.mx, Pedro de Alba s/n, San Nicolás, México, \\ Teléfono 5537063403
}

Información del artículo revisado por pares

Fecha de aceptación: junio-2021

Fecha de publicación en línea: diciembre-2021

DOI: https://doi.org/10.29105/vtga7.1-145

\section{Resumen}

La pandemia que ha sufrido la humanidad desde el 2019 hasta la fecha, ha afectado a todas las industrias a nivel global, especialmente a las franquicias dando a este modelo de negocios un golpe fuerte en toda su extensión. En este artículo se pretende explorar las diversas aproximaciones que se han tomado para resolver los problemas operativos que estas situaciones presentan tanto para los franquiciatarios como los franquiciantes, y en base a estas situaciones por analogía poder ofrecer alternativas de resurgimiento a las empresas que se encuentren en crisis en la industria de las franquicias

Palabras clave: Franquicias, Pandemias, Adaptación, Covid-19.

\section{1.- INTRODUCCIÓN:}

La actual crisis sanitaria generada por Coronavirus Covid-SARs-19, en adelante Covid, (COVID-19) que fue reconocido por primera vez en Wuhan (China) el 31 de diciembre de 2019 (OMS,2020), en México el primer caso detectado de COVID19 fue en 27 de febrero de 2020 en la Ciudad de México. (Suárez, Suarez Quezada, Oros, \& Ronquillo De Jesús, 2020)

El mundo ha sufrido la crisis sanitaria producida por el virus Covid19, así

\begin{abstract}
The pandemic that humanity has suffered, from 2019 until now, has affected all industries globally, especially franchises, producing in this business model a strong blow in its entirety. This article aims to explore the various approaches that have been taken to solve operations that these situations present for both franchisees and franchisors, and based on these situations by analogy to be able to offer resurgence alternatives to companies that are in crisis in the franchise industry
\end{abstract}

Keywords: Franchises, Pandemic, Adaptation, Covid-19.

JEL: M10; M20; M30.

consecuentemente las empresas a nivel global se han visto gravemente afectadas, sin embargo, la manera que han abordado los problemas que ha generado esta crisis sanitaria, principalmente en el área de la economía, han sido diferentes, con resultados diversos.

Este artículo considerará algunas de estas acciones, en particular las exitosas con objeto de evaluar su aplicación para el resurgimiento de las empresas en México, particularmente en Nuevo León., para lo cual 
se explorará como el modelo de franquicia puede ser una base para este desarrollo.

La franquicia como modelo de negocios presenta una oportunidad única para que las empresas puedan desarrollar sus negocios con una tasa de crecimiento rápido (Grewal, 2011). Considerando la contracción que los negocios están sufriendo debido a las condiciones de la pandemia del Covid, que ha afectado el medioambiente de los negocios a nivel global y local. El Banco Mundial ya señala que "el impacto súbito y generalizado de la pandemia del coronavirus y las medidas de suspensión de actividades que se adoptaron para contenerla han ocasionado una drástica contracción de la economía mundial, que se reducirá un 5,2 \% este año" (Banco Mundial, 2020)

Al ser una situación histórica reciente y de alto impacto, existe una variedad de artículos escritos, pero aún no una teoría clara de un camino a seguir, sino múltiples opciones, algunas de las cuales se han tomado y han errado, en estos momentos que se escribe este artículo aún no ha pasado esta pandemia y es difícil tener una visión generalista cuando se encuentra en el medio de la tormenta y aún no se ha salido de ella, lo que dificulta su evaluación y la medición real de los daños causados.

Algunos de los límites que tiene esta investigación descriptiva es el período de tiempo breve de menos de un año de la pandemia y el impacto profundo que ha causado en el modelo de las franquicias así como en el conjunto empresarial, algunas empresas han podido llevar esta situación mejor que otras gracias a la rapidez y flexibilidad de adaptación a las nuevas circunstancias, mientras que otras, algunas de las cuales se consideraban gigantes de la industria, prácticamente han desaparecido o tienen que realizar grandes esfuerzos económicos financieros para no desaparecer del todo. Por las características de la pandemia las más afectadas serán aquellas cuyo modelo de negocio contaba con pocos canales de distribución, concentrados, con características de reunión de grupos amplios de personas y que estos productos y servicios no se puedan redistribuir a domicilio de las personas y clientes, tales como los cines y espectáculos de entretenimiento. Otra industria que se ve altamente impactada negativamente es la industria inmobiliaria por su efecto en la desocupación de locales comerciales, así como de oficinas. Por otra parte, esta misma situación ha beneficiado a algunas empresas e industrias. La industria de la computación y telecomunicaciones ha sufrido un rápido incrementos, productos que se encontraban en un ciclo de vida de producto en declinación como los equipos de cómputo desktop de oficina rápidamente incrementaron sus ventas por los requerimientos de las nuevas condiciones de los consumidores, así también todas las tiendas y comercio que ofrecían sus productos por los canales digitales, o que fueron capaces de adaptarse prontamente a estos canales. Otra industria que emergió fuertemente fue el área de la logística y distribución, especialmente en el concepto de Delivery o última milla, orientadas a la distribución de bienes de consumo

$\begin{array}{cccc}\text { Este } & \text { artículo } & \text { pretende } & \text { recabar } \\ \text { información } & \text { sobre } & \text { las } & \text { diferentes }\end{array}$ aproximaciones que han tenido empresas e industrias ante esta situación de tal manera ver cuáles de ellas han sido en algún grado exitosas y cuales han tenido un fracaso. De tal forma aportar al conocimiento $y$ a las empresas franquiciantes en México y en particular en Nuevo León alternativas para mantener y desarrollar sus modelos de negocio de franquicia considerando que este modelo, ofrece grandes ventajas para resurgir luego de una crisis inesperada como esta. 


\section{2.- MARCO TEORICO:}

\section{ANTECEDENTES DEL PROBLEMA}

Con base a lo informado por INEGI (Instituto Nacional de Estadísticas Geografía e Información) señala que más de un millón de empresas cerraran o entraran en quiebra debido a los efectos de la pandemia.

Según INEGI en 15 meses se fundaron 619 mil 433 unidades económicas nuevas, lo que genera un total entre empresas que cesaron operaciones y aquellas que se agregaron a la estructura productiva de México, hay $391 \mathrm{mil}$ 414 unidades menos, es decir un 8.06 por ciento menos que en mayo de 2019. Lo que implica una reducción real de 2 millones 885 espacios de trabajo en ellas. (Inegi, 2020)

Entre 2012 y 2020 la tasa mensual de creación de empresas se mantuvo sin cambios importantes, entre 0.57 por ciento a 0.59 por ciento; pero el de negocios que dejaron de operar se duplicó, al pasar de 0.71 por ciento registrado hace ocho años a 1.36 por ciento actual (Periódico La Jornada, 2020)

Algunos de los efectos más importantes que se indican son; la disminución de ingresos, baja demanda, escasez de insumos y materias primas productivas. También se redujo la cantidad de empresas con acceso a apoyos de gobierno, lo cual pasó de 7.8 en agosto 5.9 por ciento. (Inegi, 2020)

El 15.8 por ciento de las empresas estima que no podrá continuar sus operaciones de continuar la situación actual, y no más de tres meses, de continuar con la baja de ingresos operacionales que esto genera. Un 46.6 por ciento estima que podría sostenerse un máximo 12 meses (El Financiero, 2021).
Los sectores más afectados son aquellos donde la producción de bienes y servicios está altamente relacionada con el contacto personal directo con los consumidores.

El efecto que está teniendo la pandemia también es materia de estudios a nivel global. En el trabajo de investigación realizado por Ernesto López Córdova, economista del Banco Mundial, y que considero más de 100 mil empresas en 51 países observo una mayor afectación en aquellas áreas industriales que requerían una interacción cara a cara, así también como en los trabajadores de menores ingresos y en las unidades económicas operadas por mujeres. (Cirera, y otros, 2021) . Se encontró que las ventas en las micro y pequeñas empresas cayeron en más de 50 por ciento con relación a su situación antes de la crisis sanitaria. Por otra parte, las grandes empresas la caída ha sido de alrededor de 40 por ciento. Como una respuesta a esta situación cerca del 34 por ciento de los negocios ha incrementado el uso de plataformas digitales; y más de un 17 por ciento en inversión en soluciones digitales; 21 por ciento en innovación de nuevos productos y 5 por ciento a optado por producir insumos para salud, todo esto a nivel global.

También a partir de la pandemia se puede observar que las micro y pequeñas empresas se encuentran ya en mora o estiman entrar en cesación de pago en los próximos seis meses. Esto es preocupante ya que en la medida que la crisis se prolongue y con ella los problemas financieros que lleven a la pérdida de empleos, puede causar problemas de largo plazo a las economías nacionales y disminuir su capacidad de recuperación futura según lo indicado por Córdova.

\section{OBJETIVO GENERAL}

Generar propuestas en base al modelo de negocios basado en el desarrollo de 
franquicias para apoyar a las empresas, tanto franquiciantes como franquiciatarios a volver a una situación igual o superior a la que se tenía con anterioridad a la situación generada por el SARs Covid-19.

\section{OBJETIVOS ESPECÍFICOS}

Revisar bibliografía sobre las franquicias y la pandemia y verificar diferentes aproximaciones que han realizado las empresas y organismos ante la situación de pandemia.

Verificar mediante la investigación bibliográfica las alternativas que ofrece el modelo de franquicias para lograr una recuperación a niveles similares al del 2019 o superiores.

Las franquicias al igual que otras empresas se ven afectadas de manera similar por los efectos en la economía nacional, sin embargo, con sus características especiales que las diferencian de los negocios no integrados vertical $\mathrm{u}$ horizontalmente, tiene mayor flexibilidad para cierre y apertura de sucursales.

Proponer las bases de propuestas para el desarrollo de las empresas en crisis debido a la pandemia bajo el modelo de franquicias.

\section{3.- METODO}

El método usado para analizar el tema se realizó mediante una investigación bibliográfica documental sobre artículos publicados con relación a la crisis sanitaria y las acciones que se han tomado con relación a su efecto las empresas.

Mediante el estudio sistematizado de artículos académicos, trabajos e investigaciones realizadas previamente, se pretende poder aportar un camino a la recuperación comercial mediante el uso de las franquicias.

\section{PLANTEAMIENTO DEL PROBLEMA}

La reducción de ingresos en las empresas generados por la pandemia del Covid19, así como los problemas de preparación y planificación han generado en la mayoría de las empresas reducciones importantes de sus ingresos operacionales, lo que ha generado que muchas de ellas entren en procesos de quiebra y concursos mercantiles, así como una reducción del tamaño de las mismas que logren sobrevivir estos efectos, por esto es importante generar herramientas que permitan a dichas empresas retomar sus niveles operacionales luego de finalizada la pandemia, por esto el modelo de franquicias podría ofrecer una oportunidad para ello.

\section{4.- RESULTADOS}

A pesar de que el efecto del virus y la crisis sanitaria y económica que ha generado, se puede visualizar acciones tomadas por los diferentes gobiernos y las empresas. Es en este momento, que se necesita una intervención tanto sanitaria como económica de los Estados, ya que la iniciativa privada por sí misma no es capaz de retomar la situación anterior a la crisis de manera individual.

Hoy en día, ya a más de un año del inicio de la pandemia y cuando ya se han descubierto y comienzan a aplicarse vacunas para proteger a la población global, se pueden observar que varios autores han analizado los temas de las franquicias, desde los diferentes ángulos investigativos, entre ellos se puede mencionar el aporte realizado por Gustavo Ordoqui (2020) quien señala que se debe considerar al coronavirus no solo como una amenaza, sino también como una oportunidad 
para revisar la rutina de nuestras organizaciones.

Las enseñanzas que debe dejar esta crisis sanitaria deben de ser tanto en aspectos éticos, como jurídicos. La sociedad y el mundo de los negocios tiene que aprender de estas circunstancias y de su resolución para presentar propuestas nuevas, más o menos precisas en relación con los efectos de la crisis sanitaria.

Se puede comprender que la relación entre franquiciante y franquiciatario que es mutuamente dependiente, y deben apoyarse cada uno en su función para poder sobrevivir y superar este período de incertidumbre global $\mathrm{y}$ ajustarse a las nuevas condiciones del mercado que emergerán cuando la tasa de la enfermedad se vea reducida.

Algunas investigaciones como las realizadas por Delane Botelho y Leandro Guissoni (2016) han concluido que en el modelo de franquicia existe una fuerte relación entre el nivel de rentabilidad y el crecimiento, por lo que podría ser usada para recuperar niveles de operación de las empresas anteriores a la recesión causada por la pandemia del Covid.

Estos autores señalan que el efecto de la calidad de la relación en el desempeño financiero es más fuerte en las relaciones a largo plazo que en los de corta duración.

Por otra parte Cintya Lanchimba y Daniela Medina, en su trabajo investigativo del 2018 sobre el impacto que tienen las franquicias en el desarrollo de un país, concluyen entre otros aspectos que las franquicias son herramientas que permiten el crecimiento de los países, el estudio que ellas realizaron se centró en México, investigación que las llevo a concluir entre otros que: "la importancia de empezar a relacionar el punto de vista macroeconómico con el microeconómico, pues nos ofrece una mejor perspectiva de cómo funciona tanto la economía, la política y la sociedad" (Lanchimba \& Medina, 2018).

Otros autores han investigado estos hechos en Brasil y se considera, dada la similitud de los mercados brasilero y mexicano, así como el manejo de la pandemia que ambos países han realizado. En el artículo publicado por Ilan Alón y Vanessa Galleti (2020) se pueden observar las similitudes y algunas acciones que se tomaron en Brasil, la iniciativa privada, para paliar y reducir los efectos de la pandemia en las franquicias, entre ellas se pueden destacar algunas prácticas sugeridas y tomadas por la industria de franquicias en Brasil han sido:

1. Creación de un comité de crisis multidisciplinario

2. Evaluación de su presupuesto, preservar el flujo de caja siempre que sea posible y reducir los gastos no esenciales

3. Comprender los paquetes de ayuda de emergencia disponibles

4. Conectarse con asociaciones comerciales y sectoriales relevantes

5. Ponerse en contacto con proveedores y propietarios para renegociar contratos y reducir pérdidas.

6. Modificar los modelos comerciales existentes para mantener los ingresos.

7. Invertir y construir sobre la relación franquiciador-franquiciado

8. Invertir en comunicación y transparencia con los empleados

9. Adaptar y mejorar la comunicación con los clientes

10. Hay que considerar que la situación puede cambiar rápidamente; y será necesario reevaluar regularmente las estrategias (Deloitte, 2020) 
CEPAL por su parte ha generado algunas recomendaciones a los Estados miembros tales como:

1. Incrementar los plazos de líneas de apoyo con relación a la generación de liquidez y apoyo financiero para las empresas.

2. Apoyar a empresas de sectores que afecten de manera estrategia a la sociedad y que se vean afectadas por la crisis sanitaria.

Este conjunto de acciones implicaría un incremento de liquidez de las empresas, en particular aquellas más pequeñas, mediante:

- El aplazamiento de pagos de impuestos, cargas sociales y previsionales, así como prediales, y el anticipo de devoluciones de impuestos a lo menos hasta finales del año 2020.

- Suspensión de pagos de los servicios básicos eléctricos y de agua, además de servicios de gas y telecomunicaciones, sin recargos y sin corte de servicios, hasta finales de 2020.

- Ajuste de la flexibilidad de las condiciones de crédito:

- Períodos de gracia para créditos, de más de un año y plazos de hasta cinco años o más.

- Reforzar las operaciones de crédito mediante la banca de desarrollo, que tiene una mayor capacidad de asumir el riesgo que se asocia a las empresas de menor tamaño.

Otro conjunto de medidas que se centra en evitar la destrucción de los recursos y capacidades productivas:

- Mediante apoyo al financiamiento de la nómina salarial diferenciando según el tamaño y tipo de empresas: "30\% para las grandes empresas, $50 \%$ para las medianas, $60 \%$ para las pequeñas y $80 \%$ para las microempresas" (CEPAL, 2020).

Este subsidio ofrecerse durante un periodo de seis meses.
Realizar un apoyo en efectivo para los trabajadores independientes. Por un periodo mínimo de seis meses a cada trabajador independiente que CEPAL sugiere "debe llegar a lo menos hasta 500 dólares" (CEPAL, 2021).

Un último grupo de acciones debe considerar la importancia de las grandes empresas en la actividad económica nacional y su impacto en la sociedad, el empleo y el comercio internacional, ya que son protagonistas en las áreas industriales de mayor intensidad tecnológica y muy importantes en la creación de las cadenas de proveedores. Por esto el aporte al crecimiento de la productividad de los países es un elemento fundamental para lograr un crecimiento sostenido, y como un elemento estructural fundamental para lograr un modelo de desarrollo sostenible.

A pesar de que su capacidad de enfrentar de manera exitosa esta situación que produce en las organizaciones y su personal, un elevado nivel de estrés, además poder adaptarse a estas nuevas circunstancias, es mayor en la fase de emergencia, en comparación con las empresas pequeñas, las pérdidas de empleo, competencia productiva, tecnológica y exportadora son algunos de los riesgos reales que pueden afectar gravemente la reactivación posterior a la crisis sanitaria.

Con estas consideraciones se puede comprender la importancia que el Estado como un organismo representativo de la Nación y su pueblo, participe en el fortalecimiento económico de las grandes, medianas y pequeñas empresas de sectores estratégicos para el desarrollo del país.

Esto considerando no solo la importancia económica de las empresas, sino también el impacto que estas tienen en el tejido social de la nación, y así evitar o reducir las 
posibilidades de estallidos sociales que se podrían generar.

Los apoyos del Estado deben considerar que estas empresas no reduzcan su plantilla de trabajadores por un periodo de tiempo determinado a partir de la recepción del financiamiento estatal o de los aportes de capital, así como no distribuir dividendos mientras se encuentren en contingencia y con apoyos gubernamentales y aplicar programas y acciones que permitan generar la inversión, logrando así acelerar la reactivación y aumentar la sostenibilidad ambiental de sus operaciones. "Las grandes empresas deben esforzarse en reducir los plazos de pago a sus proveedores, llegando posiblemente a pagos al contado". (CEPAL, 2020)

Otra manera de adaptarse a la situación de la crisis sanitaria en la cual se ve afectada la industria de las franquicias es la terminación de los contratos por parte de los franquiciatarios, con causa de fuerza mayor, en este sentido la legislación argentina, ha introducido un concepto de Frustración, como elemento de derecho que podría optar por esta salida, tal como lo señala Fulvio Santarelli indicando:

"En Argentina tenemos la frustración del fin a partir del Código Civil y Comercial del 2015. Regula específicamente la frustración del fin del contrato como un instrumento de readecuación contractual. Esta figura también posee otros efectos, como la resolución del contrato, pero fundamentalmente busca ser un instrumento que permita la readecuación contractual". (Santarelli, 2020)
Por su parte la consultora internacional Deloitte en su sitio web corporativo y mediante un artículo de su redacción recomienda 10 medidas para poder afrontar la pandemia de forma exitosa, entre estas se puede destacar el concepto de guardar para más adelante, donde implica llevar a cabo una buena gestión empresarial y, principalmente, garantizando la seguridad, así como una organización adecuada de todos sus empleados. Ya que las empresas se están enfrentando a diferentes riesgos tanto estratégicos como operacionales, tales como el retraso o la interrupción del suministro de materias primas e insumos; posibles cambios en la estructura de demanda de los clientes; el incremento de los costos; la interrupción de las redes logísticas que a su vez provocan retrasos en entregas; también los problemas de protección de la salud y la seguridad de los trabajadores; la insuficiencia de mano de obra; o las dificultades relacionadas con el comercio de importación y exportación. Para esto Deloitte propone las siguientes medidas:

1. Establecer equipos de toma de decisiones de emergencia.

2. Evaluar los riesgos y establecer los mecanismos de respuesta de emergencia

3. Establecer un mecanismo de comunicación de información positiva para empleados, clientes y proveedores, y crear documentos de comunicación estandarizados.

4. Mantener el bienestar físico y mental de los empleados

5. Centrarse en los planes de respuesta por los riesgos generados en la cadena de suministro.

6. Desarrollar soluciones para los riesgos de cumplimiento y mantenimiento de 
las relaciones con los clientes que surgen de la incapacidad de reanudar la producción a corto plazo.

7. Responsabilidad social y estrategias de desarrollo sostenible en la toma de decisiones.

8. Elaborar un plan de gestión de los datos de los empleados, la seguridad de la información y la privacidad.

9. Las empresas deben considerar la posibilidad de ajustar sus presupuestos y sus planes de ejecución.

10. Actualizar los mecanismos de gestión de riesgos de la empresa.

(Deloitte, 2020)

En las empresas, la pandemia ha obligado a cambiar la seguridad sanitaria para sus colaboradores, proveedores, clientes y consumidores, y también la forma en que estas se relacionan entre sí. Las nuevas acciones que se han debido tomar y que han generado el distanciamiento social, han acelerado la transformación digital la cual ya se encontraba en desarrollo, y a su vez se han acelerado los procesos que buscan aumentos de la productividad y efectividad. En este entorno de cambios rápidos y de marcada incertidumbre, que se desenvuelve en función de la pandemia y crisis sanitaria generada por el Covid-19 y sus variantes, las empresas se han visto obligadas a innovar, y reevaluar la forma en que operan, cambiar y adaptar sus modelos de negocio. Esto implica generar capacidades para identificar rápidamente los cambios y evoluciones en las preferencias y deseos de los consumidores individuales e industriales y así poder garantizar que la cadena de proveedores y los servicios ofrecidos sean consecuentes con esos cambios dinámicos. Asimismo, significa incorporar la idea de que la seguridad sanitaria puede llegar a ser un factor importante para mejorar productos y servicios. (CEPAL, 2020)

La adaptación de tecnologías digitales es esencial en cualquier nuevo modelo de negocios que permita la operación de las empresas. Las funciones o áreas de la organización en las cuales esta adaptación ocurrirá inicialmente serán en las areas mercadotecnia por el contacto de estas con los consumidores y clientes, actividades tales como la promoción, ventas y entrega de bienes y servicios, así como en la interacción con los proveedores. Por otra parte, las empresas tendrán que incorporar, así como desarrollar capacidades, para adquirir y procesar las grandes cantidades de datos que apoyen en la generación de información o macrodatos para que sus procesos de toma de decisiones, de seguimiento y adaptación a los cambios en la demanda, pero también la redefinición de las cadenas de suministro. Por esto es probable que se genere una mayor incorporación de dispositivos de interconexión digital en todos los procesos productivos, así como un incremento significativo en el uso de la robótica para incrementar la eficiencia, sobre todo tomando en cuenta que las necesidades de seguridad sanitaria pueden llevar a emplear una menor cantidad de trabajadores en algunas fases de producción.

Ante esto se pueden observar algunas medidas que han tomado diferentes países con el objeto de evitar la destrucción de valor de sus economías, y estar en una posición más fuerte una vez que se logre controlar la crisis sanitaria

Algunos países han procedido a suspender los pagos de hipotecas, así como también de algunos impuestos. También en algunos países, los gobiernos han aplicado algunas acciones y medidas extraordinarias, 
con el objeto de reducir los efectos económicos y sociales que provoca la pandemia con su crisis sanitaria.

Para el caso en Italia, puso en práctica un conjunto de acciones de apoyo que consideraron entre otros aspectos suspender el pago de diferentes impuestos así como las hipotecas con lo que se redujo la carga financiera y presión sobre empresas y los hogares.

Con relación a Estados Unidos redujo su tasa de interés, que ya se encontraba en mínimos históricos, ha lanzado programas de estímulo por más de US\$700.000 millones y luego un estímulo de 1.9 billones de dólares en un intento por proteger su economía. Además de comenzar a preparar un ambicioso programa de reactivación económica mediante el desarrollo de infraestructura federal.

Otros países han considerado relajar la política fiscal con el objetivo principal de apoyar a quienes resulten más afectados por la importante caída de la actividad económica.

Las aerolíneas ya informaron consideraban que las pérdidas durante el año llegarían a más de US\$113.000 millones en ingresos del 2020.

Uno de los sectores más golpeados ha sido el turismo internacional, con aerolíneas, hoteles y agencias de viaje pasando por uno de sus peores momentos. Los que se espera repunten significativamente una vez que se alcanza un nivel de protección antiviral alto en la población, y como efecto de las restricciones que han tenido las personas durante este año.

Algunas medidas económicas se han implementado en diversos países con el objetivo de ayudar a sus ciudadanos y empresas más afectadas por el coronavirus, durante esta crisis.
1. Suspensión de pagos de hipotecas, en este caso Italia suspendió el pago de hipotecas a las familias afectadas por el coronavirus, dicha prorroga podría llegar hasta los 18 meses y al momento de reinicio de los pagos, estos seguirán bajo las mismas condiciones que tenían antes de la implementación de la moratoria o periodo de gracia.

2. Posponer el pago de impuestos por unos meses, en el caso de España, se estableció una gracia de hasta seis meses para el pago de impuestos a pequeñas y medianas empresas (pymes) así como a los trabajadores independientes, con una bonificación en los intereses.

3. Generar préstamos a las empresas. En Alemania el estado se comprometió a generar liquidez ilimitada a las empresas para protegerlas frente a los efectos de la pandemia.

Mediante un programa de garantías de la banca pública para créditos a las empresas, sin un tope máximo.

En el caso de España, este país dispuso una línea de financiamiento especial a través del Instituto de Crédito Oficial para servir, ante los requerimientos de liquidez de las empresas y trabajadores independientes especialmente del sector turístico, así como otras areas o industrias que se estén viendo afectadas por la pandemia y crisis sanitaria.

Francia estableció la posibilidad que el estado garantice hasta el $70 \%$ del crédito a las pequeñas y medianas empresas que tengan problemas para obtener créditos bancarios y España también ofreció créditos a los sectores más afectados.

4. Rebaja de las cotizaciones sociales

Por su parte en estados unidos, la casa blanca propuso reducir las cotizaciones sociales temporalmente para que la renta neta de los trabajadores después de impuestos y retenciones sea mayor. 
5. Financiamiento de la reducción de horas de trabajo

En Alemania se aprobó un programa de apoyo a las empresas que requieran reducir las horas trabajadas.

El sistema alemán establece que la agencia de empleo estatal financiara hasta el $60 \%$ de la pérdida neta de salario causada por la reducción de horas trabajadas. En el nuevo escenario de emergencia, ese sistema se amplía.

En Italia se determinó entregar apoyos en forma de permisos laborales para los trabajadores con hijos a su cargo. o un bono cercano a los US $\$ 660$ para las personas que no puedan trabajar desde la casa y deban contratar a una persona que cuide a los niños.

Francia también genero los recursos para que los ciudadanos que lo requieran puedan ajustar su jornada de trabajo.

Entre los retos a los que se enfrentan los emprendedores se señala el aspecto de la digitalización de sus organizaciones, debido a que, al estar las personas confinadas, o con movilidad reducida, muchos productos $\mathrm{y}$ servicios además de los tratos entre empresas y clientes se deben hacer mediante diferentes plataformas de videoconferencias.

Un desafío importante está relacionado con el financiamiento de los emprendedores y sus empresas, ya que resulta claro que si no venden no se puede tener ingresos, a pesar de esto, es destacable el esfuerzo realizado por algunos gobiernos para poder apoyar a los emprendedores a superar estos desafíos.

Otro elemento importante para considerar es la percepción de oportunidades, no obstante, sobre esto hay que indicar que la literatura ha identificado "los periodos de crisis como el momento idóneo para que los individuos puedan encontrar oportunidades innovadoras." (Nicolás Martíneza \& Rubio Bañón, 2020)
Se debe destacar el desafío que representa el capital humano, ya que muchas empresas han detectado que existía falta de trabajadores (como es el caso del sector salud), mientras que en su opuesto se encuentran en la mayoría de las empresas que, al tener que cerrar sus establecimientos por tener exceso de personal para sus operaciones.

Todo esto hace pensar que resulta necesario diseñar unas nuevas líneas de operación del recurso humano y su gestión, que sean apropiadas al actual, complejo e incierto contexto en el que nos encontramos. Para ello, se debe hacer uso de todos los recursos disponibles y, con ellos, se puede impulsar a los individuos a identificar y explotar oportunidades de negocio. Los resultados de los diversos estudios realizados e inclusive la información oficial de las autoridades gubernamentales muestran, una fuerte reducción de la actividad empresarial. Se puede considerar que está afectada directamente por los niveles de incertidumbre ante un escenario nuevo y desconocido para todos. La antigüedad de la empresa y el hecho de tener trabajadores contratados ha favorecido la normalidad en aquellas empresas que no han tenido que cerrar por orden administrativa. La formación del gerente y del directivo también ha sido clave para la continuidad de la empresa. La velocidad e intensidad de los cambios aumenta la importancia de las habilidades, conocimientos y experiencias del directivo en todo tipo de empresas. "La ausencia de capacidad en el directivo es una de las principales causas de fracaso en las pequeñas y medianas empresas" (Ahire \& Golphar, 1996).

Sin duda, el cambio más relevante en el ámbito laboral es el teletrabajo, muchos de los trabajadores han estado trabajando de forma remota desde sus domicilios. Una opción que, antes de la crisis sanitaria, solía constituir una rareza o excepción (Nicolás Martíneza \& 
Rubio Bañón, 2020) Tanto es así que la Ley Federal de Trabajo mexicana, en cuya modificación del 11 de Enero del 2021, se incorporan en el capítulo 12 Bis, articulo 330A, la que se debió modificar para adecuarse a estas situaciones y definir claramente el concepto de teletrabajo, así como la responsabilidad de los patrones y trabajadores en esta modalidad de trabajo.

Existe una clara problemática, riesgos y oportunidades generales de las empresas de superar los desafíos que se plantean debido a la pandemia, con relación a su operación. En términos generales ninguna empresa estaba preparada para los efectos que genero la pandemia, sin embargo, algunas de ellas pudieron aprovechar de mejor manera esta situación, y otras se pudieron adaptar relativamente rápido, sin embargo, una cantidad importante de empresas no se ha podido adaptar y muchas de ellas han entrado en procesos de cierres generales o quiebras definitivas.

Por esto es importante poder investigar $\mathrm{y}$ analizar, que alternativas las empresas pueden aplicar para que en base a buenas decisiones en periodos de crisis puedan lograr sobrevivir en el largo plazo, en particular mediante el uso del modelo de franquicias.

El modelo de franquicias ofrece esta flexibilidad, tanto en su acciones de ajuste por reducción, en el sentido que los franquiciatarios pueden cerrar negocios individuales debido a esta situación y que sus efectos son menores al caso de una empresa de desarrollo basada en una integración vertical total, por otro lado aquellos empresarios que deseen volver a emprender luego de tener que cerrar sus operaciones, las franquicias ofrecen la posibilidad de retornar a los negocios en base a la experiencia previa que tiene el franquiciante lo que acelera su crecimiento.
Por parte del franquiciante, debe concentrarse en el desarrollo en el largo plazo, es fundamental que se adapte rápidamente a la nueva realidad y a los desafíos que se encontrarán en el desarrollo de esta crisis sanitaria originada en China, y profundizada por la incompetencia de los diferentes gobiernos, así como los cambios de los consumidores posterior a esta situación. En el corto plazo se debe ajustar en sus costos directos para tener una mejor posibilidad de sobrevivir, adicionalmente es importante el desarrollo de sus relaciones con sus franquiciatarios apoyándolos para que la mayoría de ellos puedan continuar con la franquicia. En este sentido los apoyos pueden ser económicos como es los ajustes a las regalías y otros costos, como técnicos con relación a ajustes de la oferta, y en particular las formas de distribución, acelerando la incorporación de canales digitales y servicios omnicanales tanto para franquiciatarios como consumidores finales.

\section{5.- CONCLUSIONES}

Ante la situación que se ha generado de manera extraordinaria en las empresas en general y las franquicias en el caso particular analizado se considera que existe una oportunidad de profundizar en un futuro las investigaciones, sobre los orígenes, desarrollo y acciones exitosas para sortear esta grave crisis sobre las posibilidades que tiene las diferentes empresas de sobrevivir a eventos extraordinarios que los afectan de manera inmediata.

En esta situación de crisis provocada por factores exógenos y globales, es fundamental la participación de manera importante del Estado para generar las condiciones que eviten o reduzcan posibilidades de estallidos sociales y permitir 
el desarrollo acelerado de la recuperación de la economía y la generación de empleo.

La iniciativa privada por sí sola no puede generar toda la recuperación, pero es una parte importante en la generación de empleos, pero también en su aporte a la sociedad en todas sus dimensiones.

El modelo de franquicias por ser un modelo de negocio que genera un rápido crecimiento permitiría crecer a tasas altas dándoles fuerzas tanto al franquiciante como al franquiciatario para retomar niveles de operaciones previas, también estos deben considerar las nuevas condiciones del mercado y adaptarse a ellas.

El liderazgo de las acciones e innovaciones lo debe llevar el franquiciante ya que es quien realiza la oferta de la empresa que desarrolla el modelo de negocio en base a la franquicia, y debe afectar positivamente tanto al franquiciante como al franquiciatario.

Medidas específicas que pueden aportar el modelo de franquicias para la recuperación rápida de las empresas. Desde el accionar del franquiciante, este debería otorgar al franquiciatario reducciones de costos temporales, en relación a regalías y cuotas de incorporación, negociar con proveedores de activos fijos, maquinarias y materias primas mejores condiciones económicas para los franquiciatarios, ofrecer flexibilidad en la contratación de personal y horarios de atención para ajustarse a la nueva realidad, ofrecer planes de recuperación de actividad en casos de cierres temporales por recrudecimiento de la crisis sanitaria.

Establecer una reducción de oferta de productos para concentrarse solamente en los más importantes y reducir la variedad de la oferta, así generar economías de escala. Investigar nuevas condiciones de los consumidores finales con relación a sus productos, evaluar apoyos gubernamentales de diferente índole para compartirlos con sus franquiciatarios.

El Franquiciante debería establecer un comité ad-hoc para desarrollo de planes de emergencias aplicables a la crisis sanitaria, pero también a otras situaciones posibles como serían crisis climatológicas, desastres naturales, económicos y sociales.

Incentivar un uso intensivo de tecnologías digitales de no contacto y procesos de automatización.

Generación de un grado mayor de integración vertical, y búsqueda de proveedores locales para reducir la exposición al mercado global en el corto y mediano plazo para reducir los efectos de eventuales brotes del virus que afecten las cadenas de suministros.

Un aspecto poco considerado, pero de gran importancia, es la gestión de los recursos humanos y los colaboradores, ya que la experiencia y lealtad hacia la empresa y la marca será muy difícil de recrear, por lo que el cuidado del personal, tanto del franquiciante como del franquiciatario es fundamental, en específico su salud física y mental. Para ello se debe implementar de manera definitiva el teletrabajo, en un formato híbrido que permita a los colaboradores realizar sus actividades de manera remota pero también manteniendo un contacto de baja frecuencia presencial con sus compañeros.

El conjunto de estas acciones impactaría también en el valor de la marca de la franquicia, en la percepción de los franquiciatarios, los potenciales franquiciatarios y los consumidores que es el elemento fundamental de cualquier empresa que opere bajo el modelo de franquicias, lo que permitiría con una posición fuerte incrementar el precio de las franquicias en el 
mediano plazo, así como la demanda por ella. Con estas acciones la empresa franquiciante podrá salir de la crisis sanitaria fortalecida con base a las acciones que se han tomado, y tendrá una mejor posición para tomar ventaja de las nuevas condiciones de mercado.

Los desafíos son grandes, pero así también las oportunidades, aquellas empresas que puedan emerger de la crisis sanitaria, estarán en mejor posición para aprovechar las oportunidades que se generarán en las nuevas condiciones de los mercados locales y nacionales. La franquicia por sus características específicas es uno de los modelos de negocios que permitiría esto.

Aún queda mucho espacio para continuar investigando sobre formas en que las empresas en general y en particular las mexicanas podrían emerger fortalecidas de la crisis sanitaria, lo que se presenta como un desafío para los investigadores. Algunas areas que se deberían profundizar serían las relacionadas con las nuevas conductas y características de los consumidores, las aplicaciones de nuevas tecnologías y automatización en los diferentes modelos de negocios, los efectos en la globalización o centrarse en operaciones locales nacionales de las empresas, así como evaluar la relación entre el Estado, la sociedad civil y las empresas en México y un Nuevo León postpandémico. 


\section{Referencias}

Banco Mundial. (29 de 01 de 2020). Banco Mundial. Obtenido de Banco Mundial: https://www.bancomundial.org/

BBC-Mundo. (16 de 03 de 2020). Medidas sin precedentes de países para limitar el impacto del Coronavirus. Obtenido de Medidas sin precedentes de países para limitar el impacto del Coronavirus: https://es-us.finanzas.yahoo.com/noticias/coronavirus-5-medidasecon\%C3\%B3micas-precedentes-

054151934.html?guccounter=1\&guce_referrer=aHR0cHM6Ly9ldi50dXJuaXRpbi5jb2 0v\&guce_referrer_sig=AQAAANaA2ZCk7ovvT0p2SCiX3GB3YOKWik4LUg7-

LjAXDrZv1DONyt8WvVkCLCycd4cCdy

Botelho, D. a. (2016). FRANCHISOR-FRANCHISEE RELATIONSHIP QUALITY: TIME OF RELATIONSHIP AND PERFORMANCE. RAE-Revista de Administração de Empresas |, 600-611.

CEPAL. (02 de 06 de 2020). Sectores y empresas frente al COVID-19: emergencia y reactivación. Sectores y empresas frente al COVID-19: emergencia y reactivación. santiago de Chile, Chile.

CEPAL. (15 de 01 de 2021). Comisión Económica para América Latina y el Caribe. Obtenido de Comisión Económica para América Latina y el Caribe: https://www.cepal.org/es

Cirera, X., Vargas Da Cruz, M. J., Davies, E. A., Grover, A. G., Iacovone, L., Lopez Cordova, J. E., . . . Torres, J. (13 de 01 de 2021). Policies to Support Businesses through the COVID-19 Shock : A Firm-Level Perspective (English). Policy Research working paper;no. WPS 9505;COVID-19 (Coronavirus) . Policies to Support Businesses through the COVID-19 Shock: A Firm-Level Perspective (English). Policy Research working paper;no. WPS 9505;COVID-19 (Coronavirus). Washington, DC, Estados Unidos de N.A.: World Bank Group. Obtenido de http://documents.worldbank.org/curated/en/188461610547807479/Policies-to-SupportBusinesses-through-the-COVID-19-Shock-A-Firm-Level-Perspective

Deloitte. (2020). Gestionando_el_flujodecaja_periodo de crisis. Recuperado el 2021, de Gestionando_el_flujodecaja_periododecrisis:

https://www2.deloitte.com/content/dam/Deloitte/co/Documents/risk/Gestionando_el_fl ujodecaja_periododecrisis_VR05052020.pdf

El Financiero. (30 de 01 de 2021). Periodico El Financiero. Obtenido de El Financiero: https://www.elfinanciero.com.mx/empresas/en-riesgo-de-quiebra-25-de-lasfranquicias-en-mexico-por-covid-19

Galetti Bretas, V. P., \& Alon, I. (2020). The impact of COVID-19 on franchising in emerging markets: An example from Brazil . Global Business and Organizational Excellence, 616.

Gomar Alzaga, R. d. (01 de 06 de 2020). EFECTOS DEL COVID-19 EN LA EMPRESA. Revista de Derecho La Salle, 29-56.

Grewal, D. I. (2011). Franchise partnership and international expansion: A conceptual framework and research propositions. Entrepreneurship Theory and Practice, 35(3), 533-557. doi:10.1111/j.1540-6520.2011.00444.x

Guarín Araque, C. A. (2020). Análisis del impacto causado por la pandemia del covid-19 en empresas del sector comidas rápidas de Barrancabermeja (Santander), mediante el uso de herramientas de planificación estratégica. Análisis del impacto causado por la pandemia del covid-19 en empresas del sector comidas rápidas de Barrancabermeja (Santander), mediante el uso de herramientas de planificación estratégica. Barrancabermeja, Santander, Colombia: Universidad Cooperativa De Colombia, Facultad De Ingeniería, Programa De Ingeniería Industrial.

Inegi. (30 de 12 de 2020). Inegi. Obtenido de Inegi: https://www.inegi.org.mx

Játiva Pesantez, M. A., \& Cabezas Maldonado, J. L. (Septiembre-Diciembre 2020 de 2020). La Sinergasia y nuevos emprendimientos innovadores durante la pandemia del Covid 19 en Ecuador, primer semestre de 2020. Innova Research Journal, 5 (3.1), 201-215. doi:https://doi.org/10.33890/innova.v5.n3.1.2020.1530 
Lanchimba, C., \& Medina, D. (2018). Impacto del franquiciamiento en el desarrollo. Revista Problemas del Desarrollo, 193.

Lasarte, C. (2020). Incidencias contractuales del coronavirus y cláusula rebus sic stantibus. Ius et Praxis, Revista de la Facultad de Derecho(50-51), 81-87. doi:https://doi.org/10.26439/iusetpraxis2020.n50-51.5039

Nicolás Martíneza, C., \& Rubio Bañón, A. (01 de 07 de 2020). Emprendimiento en épocas de crisis: Un análisis exploratorio de los efectos de la COVID-19. Small Business International Review, 53-66. doi:https://doi.org/10.26784/sbir.v4i2.279

Ordoqui Castilla, G. (2020). El deber de renegociar el contrato de buena fe en épocas del COVID-19. Ius et Praxis, Revista de la Facultad de Derecho , 31-43.

Periódico La Jornada. (02 de 12 de 2020). La Jornada. Obtenido de La Jornada: https://www.jornada.com.mx/ultimas/economia/2020/12/02/desaparecieron-un-millon10-mil-empresas-hasta-agosto-inegi-3266.html

Sandoval, A. (17 de 07 de 2020). La "receta" de la Cepal para que Latinoamérica enfrente la crisis por COVID-19. Obtenido de La "receta" de la Cepal para que Latinoamérica enfrente la crisis por COVID-19: https://es-us.finanzas.yahoo.com/noticias/receta-cepallatinoam\%C3\%A9rica-enfrente-crisis-170000338.html

Santarelli, F. (2020). La pandemia como elemento de la frustración del fin del contrato. Ius et Praxis, Revista de la Facultad de Derecho, 113-119.

Suárez, V., Suarez Quezada, M., O. R., \& Ronquillo De Jesús, y. E. (2020). Epidemiología de COVID-19 en México: del 27 de febrero al 30 de abril de 2020. Revista Clínica Española, 463-471.

Villanueva, D. (02 de 12 de 2020). Quiebra 8\% de empresas en 15 meses, reporta Inegi. Obtenido de Quiebra $8 \%$ de empresas en 15 meses, reporta Inegi: https://www.jornada.com.mx/notas/2020/12/02/economia/desaparecieron-un-millon10-mil-empresas-hasta-agosto-inegi/ 